\title{
Regulation of NBS-LRR Genes by MicroRNAs in Wheat: Computational Identification of Candidate MIR-2118 Genes and Evidence of Flexibility
}

\author{
Y. Khalfallah ${ }^{1}$, D. Bouktila ${ }^{1,2 *}$, Y. HabachI-Houimli ${ }^{1}$ and H. MaKNI ${ }^{1,3}$ \\ ${ }^{1}$ Unité de Recherche UR11ES10 Génomique des Insectes Ravageurs des Cultures d'intérêt agronomique \\ (GIRC), Faculté des Sciences de Tunis, Université de Tunis El Manar, 2092 El Manar, Tunisia \\ ${ }^{2}$ Institut Supérieur de Biotechnologie de Béja (ISBB), Université de Jendouba, 9000 Béja, Tunisia \\ ${ }^{3}$ Institut Supérieur de l'Animation pour la Jeunesse et la Culture (ISAJC), Université de Tunis, \\ 2055 Bir El Bey, Tunisia
}

(Received 24 June 2016; Accepted 15 August 2016;

Communicated by J. Kolmer)

\begin{abstract}
Plant microRNAs are crucial for post-transcriptional regulation of gene expression, with some of them particularly involved in regulating several disease resistance $(R)$ genes. This study is a computational screening for microRNAs potentially involved in the regulation of disease resistance in bread wheat Triticum aestivum, through the genome and transcriptome of this crop species. We used plant miRNAs of the superfamily miR-482/2118 to find complementarities with the expressed sequence tags (ESTs) coding NBS-LRR proteins of the bread wheat. This analysis revealed a vast recognition potential, highlighting highly variable miRNA-mRNA hybridization schemes. Precursors of miR-2118/482 sequences that showed a potential for recognizing wheat NBS-LRR ESTs were used as input for similarity search in the T. aestivum transcriptome shotgun assemblies (TSA) database, enabling the identification of a couple of wheat TSA sequences (JV952948.1 and JV851699.1) that were predicted to adopt a stable miRNA/miRNA* duplex structure. The genomic regions corresponding to both predicted tae-miR-2118 genes were determined on wheat chromosomes 2DL and 5AS. The findings of the present study will contribute to a better understanding of $R$ gene expression regulation in wheat.
\end{abstract}

Keywords: Triticum aestivum, microRNA, NBS-LRR, miR-2118, defense genes regulation

\section{Introduction}

Identification of small non-coding RNA (sRNA) has been, through last decades, a major research field, as they are involved in gene expression regulation at the post-transcription level. These sRNA are classified into two classes: small interference RNAs (siRNA) and microRNAs (miRNA). The first miRNA identified in a plant species, was associated with Arabidopsis thaliana immunity (Llave et al. 2002). With the increasingly available plant sequencing data, identifying miRNAs and their targets, serves to improve knowledge of

*Corresponding author; E-mail: dhia_bouktila2000@yahoo.fr 
their regulatory role over a large spectrum of plant developmental functions, including hormonal signaling, growth, developmental pattern, metabolic processes and response to the stress (Jones-Rhoades and Bartel 2004; Wang et al. 2004; Zhang et al. 2005; Berezikov et al. 2006; Carthew and Sontheimer 2009). Plant miRNA genes, called MIR genes, are generally involved with protein coding genes for miRNA biogenesis. In the nucleus, long primary miRNA transcripts are produced (Jia et al. 2013), which are then processed by a Dicer-like 1 (DCL1) enzyme into the precursor miRNAs (pre-miRNAs). The latter are, subsequently, further processed into the mature miRNA/miRNA* duplex (Kidner and Martienssen 2004; Wang et al. 2004). In the cytoplasm, the duplex strands are separated, and the miRNA strand (20-24 nucleotides) makes part of an RNA-induced silencing complex (RISC), responsible for the hybridization to the 3'-untranslated region of template (target) mRNAs, leading to either inhibition of target translation or its cleavage (Kidner and Martienssen 2004; Wang et al. 2004). It has been demonstrated that several miRNA families are conserved across a large taxonomic set of plants, from mosses to angiosperms, providing evidence that the miRNA-based regulatory system spreads over 425 million years of function (Zhang et al. 2006). Particularly when studying miRNAs with a close taxonomic affiliation, cases of conservation, where a one species miRNA would have orthologs in relative species, have been frequently observed (Mendes et al. 2009). This evolutionary fact provides, therefore, a pertinent methodology of identifying new miRNA species, using similarity-based searching and annotation (Weber et al. 2005). To search homologs of known miRNAs, databases of expressed sequence tags (ESTs) provide a valuable source as they contain the greatest number of entries corresponding to true gene expression, which can be used as computational markers to isolate new expressed genes (Zhang et al. 2005).

Disease resistance genes ( $R$ genes) of the nucleotide-binding site-leucine-rich repeats (NBS-LRR) family represent the most studied $R$ genes (Ellis and Jones 2003). At protein level, the NBS domain includes highly conserved motifs which are, respectively, the Ploop, kinase-2, kinase-3a and GLPL motifs (Meyers et al. 2003). NBS-LRRs regulate defense-related responses in plants. Molecular events (e.g. reciprocal recombination, conversions and substitutions) are at the origin of the complex organization of the $R$ genes, promoting their rapid evolution, in response to the selection pressure imposed by pathogen diversification.

Several microRNA gene families are characterized by their involvement in stress-related functions and a high proportion of NBS-LRR transcripts are targeted by miRNAs, especially in the P-loop motif. The miR-482/2118 superfamily is known for its potential to control NBS-LRR genes expression (Zhai et al. 2011) and it is widely accepted that the miR482-NBS-LRR regulatory loop has evolved to counter the cost of over-expression of NBS-LRR genes in the absence of a pathogen, thus ensuring a rapid induction of disease resistance proteins upon pathogen attack (Zhu et al. 2013). The sequences of these superfamily members are variable and accessible from the miRBase (http://www.mirbase.org/). Although the miR-482 family is abundant in several plant species and could be extended to include the Arabidopsis miR-472, it has been rarely detected in monocotyledonous species (Shivaprasad et al. 2012). 
The Poaceae family accounts approximately 10,000 species, among them, the most important agricultural species worldwide is the bread wheat Triticum aestivum L., a cultivated crop across more than 240 million Ha worldwide (Curtis 2002). The modern hexaploid $(2 \mathrm{n}=6 \mathrm{x}=42$; AABBDD) bread wheat resulted from an ancient $(\sim 10,000$ years ago) hybridization event between tetraploid $(2 \mathrm{n}=4 \mathrm{x}=28$; AABB) emmer wheat (Triticum dicoccoides Körn.) and diploid ( $2 \mathrm{n}=14$; DD) goat grass (Aegilops tauschii L.). As a component of monocotyledons, Poaceae are characterized by NBS-LRR family members, belonging to the non-toll/interleukin1 receptor nucleotide binding leucine-rich repeats (non-TNL) family (Marone et al. 2013). Whereas previous studies, based on bioinformatic mining identified several miR-482 and miR-2118 family members, targeting different $R$ genes in tomato, potato, soybean and Medicago truncatula (Zhai et al. 2011; Li et al. 2012), the miRNA pool in monocotyledons remains relatively poor compared to dicotyledons. The miR-482/2118 superfamily is represented by only 38 miR-2118 variants, occurring in nine monocotyledon species with none of these variants was found in Triticum aestivum (http://www.mirbase.org/). Based upon this, the present study aimed to (1) identify potential members of this superfamily in bread wheat, and (2) analyze the recognition potential between wheat NBS-LRR transcripts and the currently available plant miR-2118/482.

\section{Materials and Methods}

\section{Raw data source}

One hundred and fifty (150) mature miRNAs of the miR-482/2118 superfamily from various plants species (52 eudicotyledons and 12 monocotyledons), found in miRBase (http://www.mirbase.org/), were downloaded separately and used as a raw dataset of our search. From these, 103 non-redundant mature miRNAs were obtained, after discarding redundant sequences.

\section{Recognition potential between wheat NBS-LRR transcripts and plant miR-2118/482}

The PsRNATarget server (http://plantgrn.noble.org/psRNATarget/; Dai and Zhao 2011) was used, with default parameters, to predict complementarities between the studied 103 plant miR-482/2118 members and the EST gene indices of the bread wheat (DFCI Gene Index version 12, April 18, 2010 release), preloaded to PsRNATarget from Unigene library, for target search. From the results, we have selected transcripts coding NBS-LRR proteins, and plant miR-482/2118 members showing complementarity to them.

Aiming to identify the recognition potential of wheat NBS-LRR transcripts by plant miR-2118/482, we have analyzed the variability inside the coding sequence of P-loop motif of the collected wheat NBS-LRR transcripts. At protein level, the different binding sites corresponding to the P-loop motif were aligned using ClustalOmega (http://www. ebi.ac.uk/Tools/services/rest/clustalo). 


\section{Homology-based screening of novel T. aestivum miR-2118/482 members}

In an attempt to identify novel wheat miRNAs of the miR-2118/482 super family, additional to 38 already available in miRBase from different monocotyledonous species, we have collected stem loop sequences from the plant miR-2118/482 mature sequences that showed a potential for recognizing wheat NBS-LRR ESTs, in the above analysis. These miR-2118/482 precursor sequences were used as input for similarity search in the T. aestivum transcriptome shotgun assemblies database (TSAdb) through the GenBank repository (http://blast.ncbi.nlm.nih.gov/), using the blastn tool, TSA as database and T. aestivum (taxid:4565) as organism. TSA sequences have been computationally assembled from cloned cDNAs, and therefore include sequences that are longer than the traditional ESTs, thus offering opportunities to detect miR-2118/482 homologs expressed by wheat.

In order to predict $\mathrm{miR} / \mathrm{miR}^{*}$ duplexes, T. aestivum TSA sequences similar to premiRNAs were submitted to the RNAfold server (http://rna.tbi.univie.ac.at/cgi-bin/RNAfold.cgi) for secondary structure analysis and thermodynamic energy-based profiling. To predict the secondary structure, this server combines separate prediction algorithms that calculate a partition function, predicts a maximum free energy (MFE) structure, and finds structures with maximum expected accuracy. Default parameters were kept for analysis.

\section{Genomic localization and gene structure prediction}

Wheat TSA accessions that were homologous to plant pre-miRNAs, and thus predicted to be encoded by MIR genes, were localized in the bread wheat genome, using The Genome Analysis Center blast server (TGAC, http://tgac-browser.tgac.ac.uk/iwgsc_css/blast.jsp). The obtained hit scaffolds were retrieved in Fasta format and submitted to FgenesH gene finding online server (http://www.softberry.com/), for MIR genes prediction.

\section{Results}

\section{Identification of a large and flexible recognition potential between wheat NBS-LRR transcripts and plant miR-2118/482}

One hundred and three (103) non-redundant mature miR-2118/482 sequences from 64 plant species were downloaded, separately from miRBase (www.miRBase.org), and used as input file in PsRNATarget. Their complementary with T. aestivum transcripts was analyzed for sequence variability of the P-loop motif. In total, we have identified various recognition patterns between miR-2118/482 and NBS-LRR mRNAs. In these patterns were involved 13 wheat NBS-LRR transcripts and 34 miR-2118/482 species from 26 different plants (Table $\mathrm{S} 1 *$ ).

Sequence analysis of wheat NBS-LRR transcripts showed several variations at the binding site to plant mRNAs, namely in the P-loop motif: GMGGV(L)GK. We have de-

\footnotetext{
*Further details about the Electronic Supplementary Material (ESM) can be found at the end of the article.
} 
fined eight different variants of the P-loop, at the protein level (Table S1). Interestingly, two different miRNAs differing by up to four nucleotides (e.g. ata-miR2118a and bdimiR2118a) could target the same NBS-LRR mRNA (e.g. NP935614) in the sequence corresponding to the P-loop motif. These observations can be explained by the relatively frequent mutational events in $M I R$ genes triggered by their birth and death evolution model (Fahlgren et al. 2007).

\section{Identification of two T. aestivum miRNAs belonging to the miR-2118 family}

Starting from the hypothesis that the 34 plant miR-2118 members would potentially have conserved counterparts among T. aestivum tae-miR-2118, we have investigated the homology between plant pre-miRNAs used as queries and T. aestivum TSA sequences. Homology searching within T. aestivum TSA sequences, using plant pre-miRNAs as queries, yielded three relevant matches among wheat TSA sequences. First, JV952948.1 (Duan et al. 2012) was matched by two pre-miRNAs of $A$. tauschii (ata-miR2118a-3p and atamiR2118b-5p) and a pre-miRNA of $B$. distachyon (bdi-miR2118b-3p), with highly significant e-values of 1e-73,1e-102 and 6e-2, respectively; secondly, JV851699.1 (Duan et al. 2012) was matched by ata-miR2118c and ata-miR2118d, with the same e-value of 1e-47; and finally, JV952923.1 (Duan et al. 2012) was matched by bdi-miR2118a, with an e-value of 4e-28. Even though it was characterized by a significant e-value, JV952923.1 was discarded from the results due to a low query-to-hit coverage (52\%). Therefore, we could consider that two sequences from the wheat TSA database (JV952948.1 and JV851699.1) corresponded to putative wheat miRNA genes.

Verification through GenBank protein database, using blastx, confirmed that both JV952948.1 and JV851699.1 did not encode any protein sequences. It is known that the miRNA ability to down regulate mRNA target, depends strongly on the pre-miRNA hairpin form. Such a structure is also important for the pre-miRNA recognition by RNAase III enzymes Drosha and Dicer, as well as for the export of the miRNA precursor from the nucleus (Lund et al. 2004). Therefore, both JV952948.1 and JV851699.1, were submitted to the RNAfold web server (http://rna.tbi.univie.ac.at/cgi-bin/RNAfold.cgi), to predict their stem loop structure. The result of this analysis, illustrated in Fig. 1, allowed making two predictions: the first, derived from JV952948.1, consisted in a putative miRNA/miRNA* duplex (5'-UUCCCGAUGCCUCCCAUUCCUA-3' and 5'-UGGGAATGGGAACATGGAGGA-3'), with an optimal secondary structure stability of $-70.90 \mathrm{kcal} /$ mol, as inferred from minimum free energy (MFE) analysis. The second predicted miRNA/miRNA* duplex, derived from JV851699.1, consisted in a putative mature miRNA/ miRNA* duplex (5'-UGGGCAUGGGAACAUGGAGGAA-3' and 5'-UUCCUGAUGCCUCCCAUGCCUA-3'), with an optimal secondary structure characterized by a minimum free energy of $-56.00 \mathrm{kcal} / \mathrm{mol}$. Both predicted wheat pre-miRNAs were labeled tae-miR2118a and tae-miR2118b (Fig. 1). 

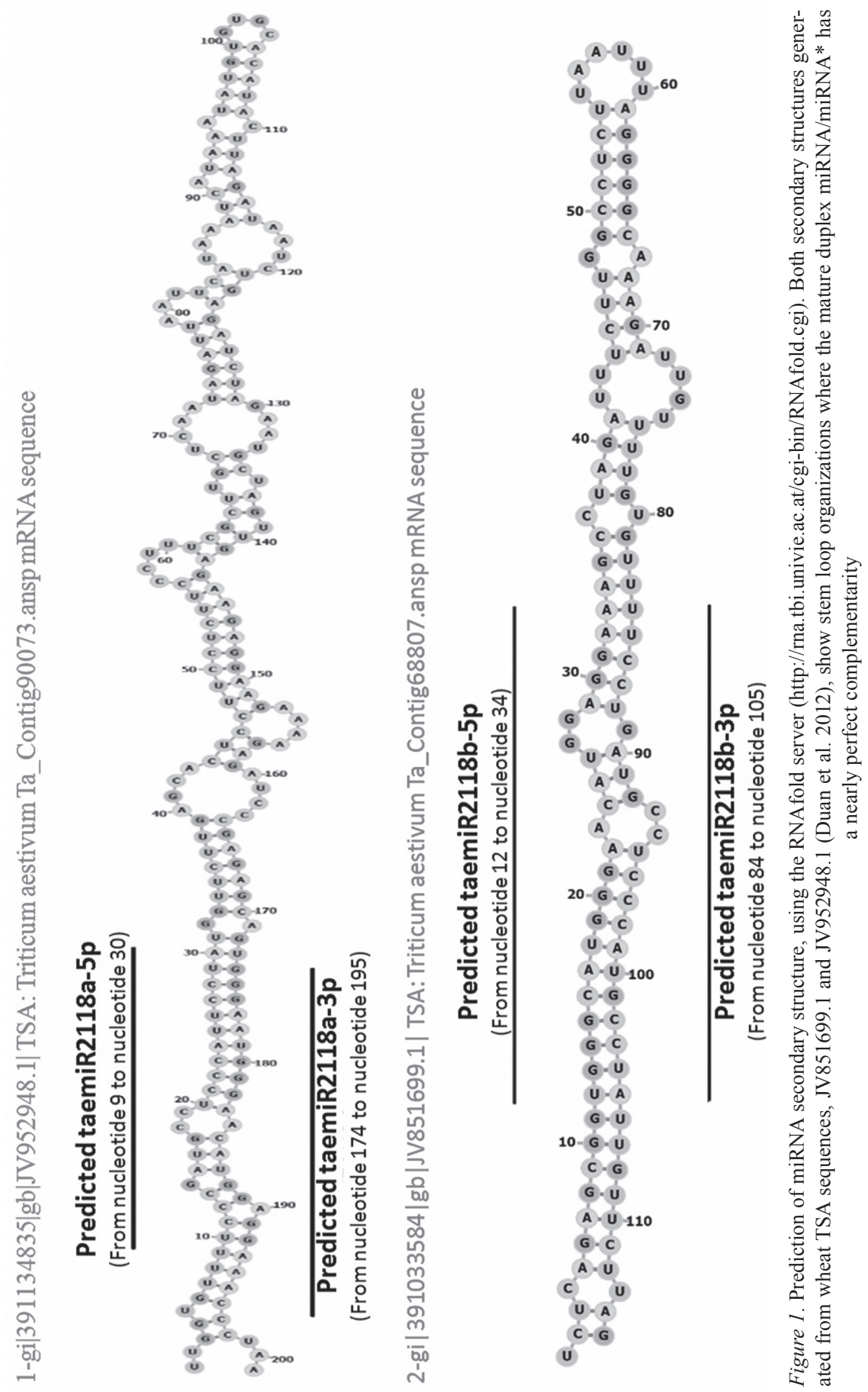

Cereal Research Communications 45, 2017 
Genomic localization and gene structure prediction of tae-miR2118a and tae-miR2118b

Both JV851699.1 and JV952948.1 were submitted to The Genome Analysis Center blast server (http://tgac-browser.tgac.ac.uk/iwgsc_css/blast.jsp) to obtain the most accurate localization in the bread wheat genome. The first TSA hit JV952948.1 matched genomic scaffold ' 9838888 ' on wheat chromosome 2DL, covering 501 bp of length, from position $6024 \mathrm{bp}$ to position $6524 \mathrm{pb}$, with $100 \%$ of identity and an expected value of 0.0 . The second TSA hit JV851699.1 matched scaffold ' 1509771 ' from position 4137 bp to position $4755 \mathrm{bp}$, on chromosome $5 \mathrm{AS}$, with $93.89 \%$ of identity with the matched genomic region and an e-value of 0.0 (Table 1). Subsequent analysis of each scaffold, separately, through FgenesH, revealed the presence of complete gene structures, where the candidate pre-miRNA sequence would be transcribed within the introns of the predicted host genes (Fig. 2).

\section{IWGSC_CSS_2DL_scaff_9838888}
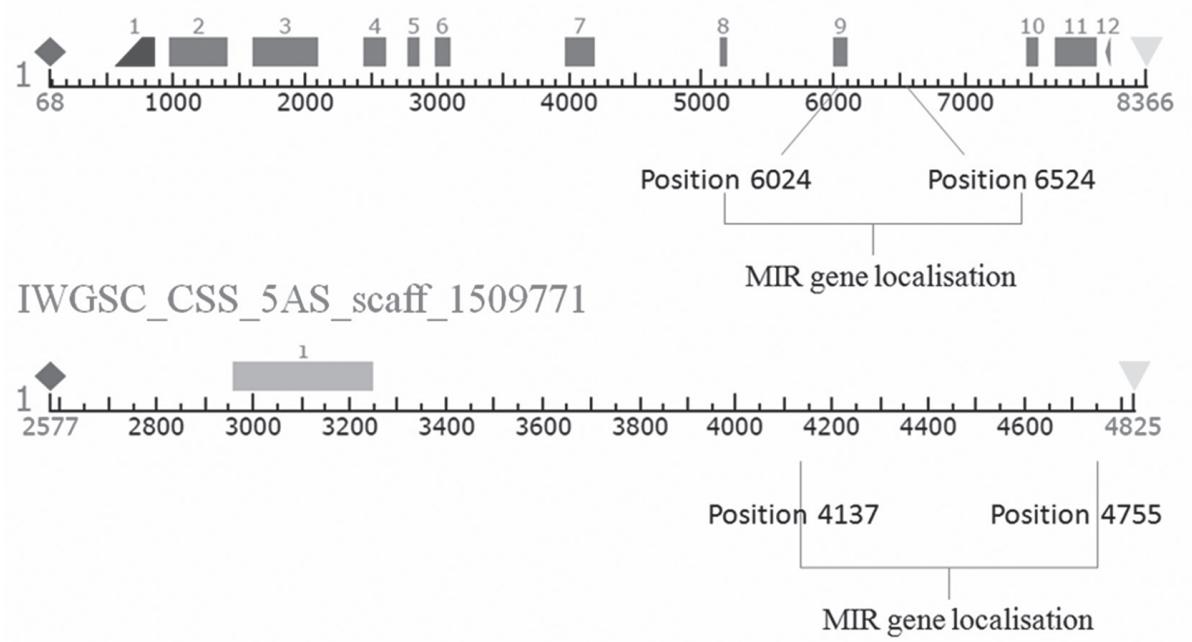

DCDf $\square \operatorname{CDSi} \mathbf{C D S I} \square \operatorname{CDSO} \gg$ PolA $\nabla$ TSS

Figure 2. Putative complete-ORF gene structures encoding tae-miR2118a and tae-miR2118b pre-miRNAs, predicted using FgenesH (http://www.softberry.com/).

CDSf: First coding segment (starting with Start codon); CDSi: Internal coding segment (internal exon); CDS1: Last coding segment (ending with Stop codon); TSS: Position of transcription start (TATA-box position and score); PolA: Polyadenylation signal sequence (AATAAA) 
Table 1. Results of BLASTn between wheat TSA sequences, JV851699.1 and JV952948.1, and

IWGSC genome survey scaffolds, accessed through TGAC's bread wheat BLAST server

\begin{tabular}{|c|c|c|c|c|c|c|c|}
\hline $\begin{array}{c}\text { Query } \\
\text { (wheat TSA) }\end{array}$ & $\begin{array}{c}\text { Subject } \\
\text { (IWGSC genome survey scaffolds) }\end{array}$ & \% identity & q.start & q.end & s.start & s.end & e-value \\
\hline JV952948.1 & IWGSC_CSS_2D_scaff_9838888 & 100 & 1 & 500 & 6024 & 6524 & 0.0 \\
\hline JV851699.1 & IWGSC_CSS_5AS_scaff_1509771 & 93.89 & 1 & 617 & 4137 & 4755 & 0.0 \\
\hline
\end{tabular}

q.start and q.end designate the query range covered by alignment; s.start and s.end designate the subject range covered by alignment.

\section{Discussion}

The aim of our study was to identify miRNAs mediating NBS-LRR genes expression in wheat. As a first step, we have identified eight different recognition patterns that involved 13 wheat NBS-LRR mRNAs with 34 miR-2118/482 sequences from different plants. We think that such flexibility is crucial to the mRNA-miRNA co-evolution, as each counterpart will have the ability to evolve few sites of mutation under selection constraints, without affecting the robustness of the regulatory process. For example in Solanaceae, such as tomato and potato, a robust targeting network within the same plant species has been recently discovered (de Vries et al. 2015). The redundancy of targeting may help to support the rapid evolution of NBS-LRRs enhanced by pathogen evolution, allowing the miR-2118 gene family to act as an evolutionary buffer for $R$ gene sequence diversity, in the context of the high selection pressure exerted reciprocally by pathogens and NBSLRR genes.

As a second step, we have identified, in silico, two T. aestivum miRNAs, tae-miR2118a and tae-miR2118b, and their predicted genes were localized on wheat chromosomes 2DL and 5AS. It is well known that plant-miRNAs are mostly generated from non-coding transcriptional units. Generally, miRNA genes origins are either from transposable elements (TE), duplication of protein coding genes, or duplication of preexisting gene followed by mutation events (Allen et al. 2005; Fahlgren et al. 2007; Fahlgren and Carrington 2010). In addition, some miRNA genes would have originated from a hairpin structure in intronic or intergenic regions ( $\mathrm{Lu}$ et al. 2008; Nozawa et al. 2010). In our study, we have found that the two predicted miRNA genes, designated tae-miR2118a and tae-miR2118b, are localized in intronic regions of protein coding genes that have perfect structural composition. Ying et al. (2010) reported that intronic miRNAs are transcribed along with primary transcript by the promoter of the host gene. Introns of protein-coding genes, where MIR genes are hosted are referred to as mirtrons. Until date, they have been discovered in invertebrates and mammals (Berezikov et al. 2007), and, more recently, in rice and Arabidopsis (Yang et al. 2012).

The findings reported in this paper will be useful to further research on miRNAs involved in disease resistance genes regulation in wheat. Our future emphasis will encompass the expression profiling of the identified miRNAs in different pathological contexts. 


\section{Acknowledgement}

This study was supported by the Tunisian Ministry of Higher Education and Scientific Research.

\section{References}

Allen, E., Xie, Z., Gustafson, A.M., Carrington, J.C. 2005. MicroRNA-directed phasing during trans-acting siRNA biogenesis in plants. Cell 121:207-221.

Berezikov, E., Cuppen, E., Plasterk, R.H.A. 2006. Approaches to microRNA discovery. Nat. Genet. 38:S2-S7.

Berezikov, E., Chung, W.J., Willis, J., Cuppen, E., Lai, E.C. 2007. Mammalian mirtron genes. Mol. Cell. 28:328-336.

Carthew, R.W., Sontheimer, E.J. 2009. Origins and mechanisms of miRNAs and siRNAs. Cell 136:642-655.

Curtis, B.C. 2002. Wheat in the World. In: Curtis, B.C. (ed.), Bread Wheat: Improvement and Production. Food and Agriculture Organisation of the United Nations (FAO). Rome, Italy. http://www.fao.org/docrep/006/ y4011e/y4011e04.htm\#bm04 (accessed 30.04.2016).

Dai, X., Zhao, P.X. 2011. PsRNATarget: a plant small RNA target analysis server. Nucleic Acids Res. 39:W155-W159.

de Vries, S., Kloesges, T., Rose, L.E. 2015. Evolutionarily dynamic, but robust, targeting of resistance genes by the miR482/2118 gene family in the Solanaceae. Genome Biol. Evol. 7:3307-3321.

Duan, J., Xia, C., Zhao, G., Jia, J., Kong, X. 2012. Optimizing de novo common wheat transcriptome assembly using short-read RNA-Seq data. BMC Genomics 13:392.

Ellis, J.G., Jones, D.A. 2003. Plant disease resistance genes. In: Ezekowitz, R.A.B., Hoffmann, J.A. (eds), Innate Immunity. Humana Press Inc. Totowa, NJ, USA. pp. 27-45.

Fahlgren, N., Howell, M.D., Kasschau, K.D., Chapman, E.J., Sullivan, C.M., Cumbie, J.S., Givan, S.A., Law, T.F., Grant, S.R., Dangl, J.L., Carrington, J.C. 2007. High-throughput sequencing of Arabidopsis microRNAs: evidence for frequent birth and death of MIRNA genes. PLoS One 2:e219.

Fahlgren, N., Carrington, J.C. 2010. Mirna target prediction in plants. Methods Mol. Biol. 592:51-57.

Jia, J., Zhao, S., Kong, X., Li, Y., Zhao, G., He, W., Appels, R., Pfeifer, M., Tao, Y., Zhang, X., Jing, R., Zhang, C., Ma, Y., Gao, L., Gao, C., Spannagl, M., Mayer, K.F.X., Li, D., Pan, S., Zheng, F., Hu, Q., Xia, X., Li, J., Liang, Q., Chen, J., Wicker, T., Gou, C., Kuang, H., He, G., Luo, Y., Keller, B., Xia, Q., Lu, P., Wang, J., Zou, H., Zhang, R., Xu, J., Gao, J., Middleton, C., Quan, Z., Liu, G., Wang, J., International Wheat Genome Sequencing Consortium, Yang, H., Liu, X., He, Z., Mao, L., Wang J. 2013. Aegilops tauschii draft genome sequence reveals a gene repertoire for wheat adaptation. Nature 496:91-95.

Jones-Rhoades, M.W., Bartel, D.P. 2004. Computational identification of plant microRNAs and their targets, including a stress-induced miRNA. Mol. Cell 14:787-799.

Kidner, C.A., Martienssen, R.A. 2004. Spatially restricted microRNA direct leaf polarity through ARGONAUTE1. Nature 428:81-84.

Li, F., Pignatta, D., Bendix, C., Brunkard, J.O., Cohn, M.M., Tung, J., Sun, H., Kumar, P., Baker, B. 2012. MicroRNA regulation of plant innate immune receptors. Proc. Natl Acad. Sci. USA 109:1790-1795.

Llave, C., Xie, Z., Kasschau, K.D., Carrington, J.C. 2002. Cleavage of scarecrow-like mRNA targets directed by a class of Arabidopsis miRNA. Science 297:2053-2056.

Lu, C., Jeong, D.H., Kulkarni, K., Pillay, M., Nobuta, K., German, R., Thatcher, S.R., Maher, C., Zhang, L., Ware, D., Liu, B., Cao, X., Meyers, B.C., Green, P.J. 2008. Genome-wide analysis for discovery of rice microRNAs reveals natural antisense microRNAs (nat-miRNAs). Proc. Natl Acad. Sci. USA 105:49514956.

Lund, E., Güttinger, S., Calado, A., Dahlberg, J.E., Kutay, U. 2004. Nuclear export of microRNA precursors. Science 303:95-98.

Marone, D., Russo, M.A., Laidò, G., De Leonardis, A.M., Mastrangelo, A.M. 2013. Plant nucleotide binding site-leucine-rich repeat (NBS-LRR) genes: active guardians in host defense responses. Int. J. Mol. Sci. 14:7302-7326. 
Mendes, N.D., Freitas, A.T., Sagot, M.F. 2009. Current tools for the identification of miRNA genes and their targets. Nucleic Acids Res. 7:2419-2433.

Meyers, B.C., Kozik, A., Griego, A., Kuang, H., Michelmore, R.W. 2003. Genome-wide analysis of NBSLRR-encoding genes in Arabidopsis. Plant Cell 15:809-834.

Nozawa, M., Miura, S., Nei, M. 2010. Origins and evolution of microRNA genes in Drosophila species. Genome Biol. Evol. 2:180-189.

Shivaprasad, P.V., Chen, H.M., Patel, K., Bond, D.M., Santos, B.A., Baulcombe, D.C. 2012. A microRNA superfamily regulates nucleotide binding site-leucine-rich repeats and other mRNAs. Plant Cell 24:859874.

Wang, X.J., Reyes, J.L., Chua, N.H., Gaasterland, T. 2004. Prediction and identification of Arabidopsis thaliana microRNAs and their mRNA targets. Genome Biol. 5:R65.

Weber, H., Polen, T., Heuveling, J., Wendisch, V.F., Hengge, R. 2005. Genome-wide analysis of the general stress response network in Escherichia coli: sigmaS-dependent genes, promoters, and sigma factor selectivity. J. Bacteriol. 187:1591-1603.

Yang, G.D., Yan, K., Wu, B.J., Wang, Y.H., Gao, Y.X., Zheng, C.C. 2012. Genomewide analysis of intronic microRNAs in rice and Arabidopsis. J. Genet. 91:313-324.

Ying, S.Y., Chang, C.P., Lin, S.L. 2010. Intron-mediated RNA interference intronic microRNAs and applications. Methods Mol. Biol. 629:205-237.

Zhai, J., Jeong, D.H., De, Paoli, E., Park, S., Rosen, B.D., Li, Y., González, A.J., Yan, Z., Kitto, S.L., Grusak, M.A., Jackson, S.A., Stacey, G., Cook, D.R., Green, P.J., Sherrier, D.J., Meyers, B.C. 2011. MicroRNAs as master regulators of the plant NB-LRR defense gene family via the production of phased, trans-acting siRNAs. Genes Dev. 25:2540-2553.

Zhang, B.H., Pan, X.P., Wang, Q.L., Cobb, G.P., Anderson, T.A. 2005. Identification and characterization of new plant microRNAs using EST analysis. Cell Res. 15:336-360.

Zhang, B., Pan, X., Cobb, G.P., Anderson, T.A. 2006. Plant microRNA: a small regulatory molecule with big impact. Dev. Biol. 289:3-16.

Zhu, Q.H., Fan, L., Liu, Y., Xu, H., Llewellyn, D., Wilson, I. 2013. MiR482 regulation of NBS-LRR defense genes during fungal pathogen infection in cotton. PLoS ONE 8:e84390.

\section{Electronic Supplementary Material (ESM)}

Electronic Supplementary Material (ESM) associated with this article can be found at the website of CRC at http://www.akademiai.com/content/120427/

Electronic Supplementary Table S1. Recognition patterns between 34 miR2118/482 species from 26 different plants and 13 wheat NBS-LRR transcripts, inferred from complementarity analysis, using PsRNATarget 